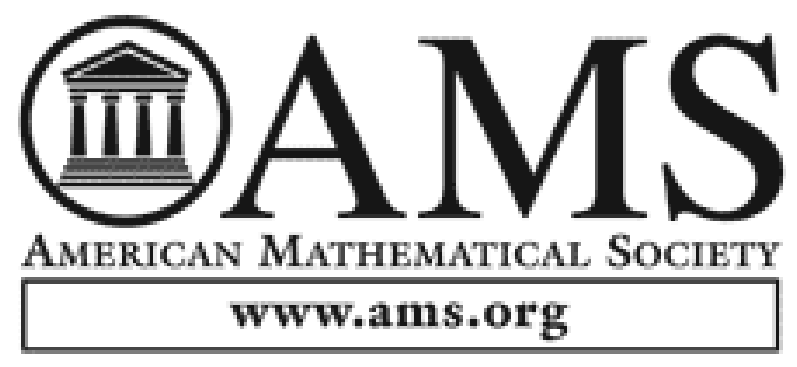

The Set of Continuous Functions with the Everywhere Convergent Fourier Series Author(s): M. Ajtai and A. S. Kechris

Source: Transactions of the American Mathematical Society, Vol. 302, No. 1 (Jul., 1987), pp. 207-221

Published by: American Mathematical Society

Stable URL: http://www.jstor.org/stable/2000906

Accessed: 20/05/2013 13:08

Your use of the JSTOR archive indicates your acceptance of the Terms \& Conditions of Use, available at http://www.jstor.org/page/info/about/policies/terms.jsp

JSTOR is a not-for-profit service that helps scholars, researchers, and students discover, use, and build upon a wide range of content in a trusted digital archive. We use information technology and tools to increase productivity and facilitate new forms of scholarship. For more information about JSTOR, please contact support@jstor.org. 


\title{
THE SET OF CONTINUOUS FUNCTIONS WITH EVERYWHERE CONVERGENT FOURIER SERIES
}

\author{
M. AJTAI AND A. S. KECHRIS
}

\begin{abstract}
This paper deals with the descriptive set theoretic properties of the class EC of continuous functions with everywhere convergent Fourier series. It is shown that this set is a complete coanalytic set in $C(T)$. A natural coanalytic rank function on EC is studied that assigns to each $f \in \mathrm{EC}$ a countable ordinal number, which measures the "complexity" of the convergence of the Fourier series of $f$. It is shown that there exist functions in EC (in fact even differentiable ones) which have arbitrarily large countable rank, so that this provides a proper hierarchy on EC with $\omega_{1}$ distinct levels.
\end{abstract}

Let $C(\mathbf{T})$ be the Banach space of continuous $2 \pi$-periodic functions on the reals with the sup norm. We study in this paper descriptive set theoretic aspects of the subset EC of $C(\mathbf{T})$ consisting of the continuous functions with everywhere convergent Fourier series.

It is easy to verify that $\mathrm{EC}$ is a coanalytic set. We show in $\S 3$ that $\mathrm{EC}$ is actually a complete coanalytic set and therefore in particular is not Borel: This answers a question in $[\mathbf{K u}]$ and provides another example of a coanalytic non-Borel set. We also discuss in $\S 2$ (see in addition the Appendix) the relations of this kind of result to the studies in the literature concerning the classification of the set of points of divergence of the Fourier series of a continuous function.

It follows also immediately that the set $\mathrm{EC}^{\prime}$ of $f \in L^{1}(\mathbf{T})$ with everywhere convergent Fourier series is (coanalytic but) not Borel (in the Banach space $L^{1}(\mathbf{T})$ of Lebesgue integrable functions on $[0,2 \pi]$ ). Equivalently working in the Banach space $c_{0}(\mathbf{Z})$ of $\mathbf{Z}$-sequences converging to 0 at infinity, the set $P^{\prime}$ (resp. $P$ ) of sequences $\left\{a_{n}\right\}_{n \in \mathbf{Z}} \in c_{0}(\mathbf{Z})$ such that $\sum a_{n} e^{i n x}$ is an everywhere convergent Fourier series (resp. of a continuous function) is also (coanalytic but) not Borel. This rules out the possibility of any "reasonable" criteria on the coefficients for characterizing when a given Fourier series $\sum a_{n} e^{i n x}$, even of a continuous function, is everywhere convergent.

By specializing a construction of Zalcwasser $[\mathbf{Z a}]$ and independently GillespieHurewicz [GH], we associate to each $f \in$ EC a countable ordinal number $|f|_{z}$ which measures the "complexity" of the convergence of the Fourier series of $f$. The functions with ordinal rank 1 are exactly the ones with uniformly convergent Fourier series. The standard examples of continuous functions with everywhere but not uniformly convergent Fourier series turn out to have rank exactly 2 . We show in $\S 4$ that the rank function $f \rightarrow|f|_{Z}$ has the right descriptive set theoretic properties summarized in the concept of a coanalytic-norm. It follows that there are

Received by the editors June 11, 1985 and, in revised form, June 20, 1986.

1980 Mathematics Subject Classification (1985 Revision). Primary 03E15, 26A21, 42A20.

Research partially supported by NSF Grant DMS-8416349. 
continuous functions whose Fourier series converge everywhere but the convergence is arbitrarily complex, i.e. for every countable ordinal number $\alpha$ there is an $f \in \mathrm{EC}$ with $|f|_{Z}>\alpha$. So this gives a proper hierarchy of all the functions with everywhere convergent Fourier series in $\omega_{1}$ levels, with functions occupying higher levels having more and more complex convergence behavior of their Fourier series.

\section{Preliminaries.}

1.1. Let $\mathbf{N}=\{1,2,3, \ldots\}$ be the set of positive integers, and $\mathbf{N}^{*}\left(\equiv \mathbf{N}^{<\mathbf{N}}\right)$ the set of all finite sequences $s=\left(a_{1}, \ldots, a_{n}\right)$ from $\mathbf{N}$ (including the empty one ()$)$. The length $n$ of $s$ is denoted by $|s|$ (thus $|()|=0$ ). An initial segment $v$ of $u$ is a sequence of the form $v=\left(a_{1}, \ldots, a_{m}\right)$, for $1 \leq m \leq n$ or $v=()$. We write also $v=u \uparrow m$ in this case $(()=u \uparrow 0)$. We call moreover $u$ an extension of $v$, and $u$ a proper extension if in addition $u \neq v$. The concatenation $u^{-} w$ of two sequences $u=\left(a_{1}, \ldots, a_{n}\right), w=\left(b_{1}, \ldots, b_{m}\right)$ is the sequence $u^{-} w=\left(a_{1}, \ldots, a_{n}, b_{1}, \ldots, b_{m}\right)$. In particular $u^{-}(a)$ is a one-element or immediate extension of $u$.

For an infinite sequence $\boldsymbol{\alpha}=(\boldsymbol{\alpha}(1), \boldsymbol{\alpha}(2), \ldots) \in \mathbf{N}^{\mathbf{N}}$ we let also $u=\boldsymbol{\alpha} \uparrow m=$ $(\boldsymbol{\alpha}(1), \ldots, \boldsymbol{\alpha}(m))$ be the initial segment of $\boldsymbol{\alpha}$ of length $m$ and we call $\boldsymbol{\alpha}$ an extension of any such $u$. We denote by $u \subset v$ or $u \subset \boldsymbol{\alpha}$ the relation of being an initial segment of. Let $u \varsubsetneqq v$ iff $u \subset v$ and $u \neq v$.

By a tree $T$ on $\mathbf{N}$ we will mean a nonempty subset of $\mathbf{N}^{*}$ closed under initial segments $(u \in T \& v \subset u \Rightarrow v \in T)$. Thus ()$\in T$. For each such tree we denote by $[T]$ the set of all its infinite branches or paths, i.e.,

$$
[T]=\left\{\boldsymbol{\alpha} \in \mathbf{N}^{\mathbf{N}}: \forall n(\boldsymbol{\alpha} \uparrow n \in T)\right\} .
$$

We call $T$ wellfounded iff $[\dot{T}]=\varnothing$.

By $2^{<\mathrm{N}}$ we denote the set of all binary finite sequences (here $2=\{0,1\}$ ) and by $2^{\mathbf{N}}$ the set of all infinite binary sequences. All the above carry over mutatis mutandis to the context of binary sequences.

1.2. A basic method for showing that a given $\Pi_{1}^{1}$ (= coanalytic) set is not Borel, is to demonstrate that it is complete in the sense of the following definition.

Definition. Let $X$ be a Polish space. $A \Pi_{1}^{1}$ subset $A$ of $X$ is called complete if for any Polish space $Y$ and any $\Pi_{1}^{1}$ subset $B$ of $Y$ there is a Borel function $f: Y \rightarrow X$ such that $B=f^{-1}[A]$.

Since for some Polish space $Y$ (e.g. $Y=\mathbf{R} \equiv$ the set of reals) there is some $B \subseteq Y$ which is $\Pi_{1}^{1}$ but not Borel, it follows that no complete $\Pi_{1}^{1}$ set is Borel.

It is clear that if $X, X^{\prime}$ are Polish spaces, $g: X \rightarrow X^{\prime}$ is a Borel function, $A \subseteq X$ is complete $\Pi_{1}^{1}$ and $A^{\prime} \subseteq X^{\prime}$ is $\Pi_{1}^{1}$ and such that $A=g^{-1}\left[A^{\prime}\right]$, then $A^{\prime}$ is also complete $\Pi_{1}^{1}$. (When $A=g^{-1}\left[A^{\prime}\right]$ as above we say that $A$ is reduced to $A^{\prime}$ via $g$. The terminology comes from the fact that $x \in A \Leftrightarrow g(x) \in A^{\prime}$, which means that the question of membership in $A$ is reduced via $g$ to that of $A^{\prime}$ ). So a standard procedure for showing the $\Pi_{1}^{1}$-completeness of a set is to reduce one of the already known $\Pi_{1}^{1}$ complete sets to it. For our purposes it is most convenient to use as our starting complete $\Pi_{1}^{1}$ set the set WF of wellfounded trees.

As a subset of $\mathbf{N}^{*}$, any tree $T$ on $\mathbf{N}$ can be identified with its characteristic function, which is a member of the Polish space $2^{\mathbf{N}^{*}}=\{0,1\}^{\mathbf{N}^{*}}$, homeomorphic to $2^{\mathbf{N}}$, i.e. the Cantor set. The set of all trees is then a closed subset of $2^{\mathbf{N}^{*}}$. Let WF be the set of all wellfounded trees on $\mathbf{N}$. Then it is a classical result that WF is a complete $\Pi_{1}^{1}$ set (see e.g. the introduction of $[\mathbf{K W}]$ ). 
1.3. A rank function or norm on a set $P$ is just a map $\varphi: P \rightarrow$ ORD from $P$ into the class of ordinals ORD. This induces a prewellordering $\leq_{\varphi}$ on $P$ defined by $x \leq_{\varphi} y \Leftrightarrow \varphi(x) \leq \varphi(y)$. We consider two norms $\varphi, \varphi^{\prime}$ on $P$ equivalent if they induce the same prewellordering $\leq_{\varphi}=\leq_{\varphi^{\prime}}$. We will be mainly interested in special norms on $\Pi_{1}^{1}$ subsets of Polish spaces.

Given a Polish space $X$ and $\Pi_{1}^{1}$ subset $P$ of $X$ we say that a norm $\varphi: P \rightarrow$ ORD is a $\Pi_{1}^{1}$-norm if there is a $\Sigma_{1}^{1}\left(=\right.$ analytic) subset $R$ of $X^{2}$ and a $\Pi_{1}^{1}$ subset $Q$ of $X^{2}$ (i.e. $R, Q$ are binary relations on $X$ ) such that

$$
y \in P \Rightarrow[x \in P \& \varphi(x) \leq \varphi(y) \Leftrightarrow R(x, y) \Leftrightarrow Q(x, y)] .
$$

This implies that the initial segments of $\leq_{\varphi}$ are Borel, but the above condition is much stronger than that. In some sense it says that the initial segments are "uniformly" Borel.

Every $\Pi_{1}^{1}$-norm is equivalent with one which takes values in $\omega_{1}=$ the first countable ordinal. This is because every proper initial segment of the induced prewellordering is Borel and Borel prewellorderings have countable ranks (see $[\mathbf{M}]$ ). It is a fundamental fact of the structure theory of $\Pi_{1}^{1}$ sets (see [M]), that every $\Pi_{1}^{1}$ set $P$ admits a $\Pi_{1}^{1}$-norm $\varphi: P \rightarrow \omega_{1}$. Such a norm can be found by means of the general theory and it is by no means unique. It is an interesting question to find for a given $\Pi_{1}^{1}$ set $P$, which arises naturally in some context in analysis, topology, etc., a natural $\Pi_{1}^{1}$-norm which reflects the properties of the set $P$ in question. We deal with this problem for the set of continuous functions with everywhere convergent Fourier series in $\S 4$.

Recall now the following basic criterion (see $[\mathbf{M}]$ ).

Given a $\Pi_{1}^{1}$ set $P \subseteq X, X$ a Polish space, and given any $\Pi_{1}^{1}$-norm $\varphi: P \rightarrow \omega_{1}$ the following are equivalent:

(i) $P$ is Borel.

(ii) $\{\varphi(x): x \in P\}$ is bounded below $\omega_{1}$.

Then in particular if $P$ is not Borel and we let for any $\alpha<\omega_{1}, P_{\alpha}=\{x \in P: \varphi(x) \leq$ $\alpha\}$ then $\left\{P_{\alpha}\right\}_{\alpha<\omega_{1}}$ is a hierarchy of $\omega_{1}$ stages on $P$. If $\varphi$ is a naturally defined $\Pi_{1-}^{1-}$ norm, this hierarchy can provide in a natural way a measure of complexity for the elements of $P$.

We conclude by noticing one further thing about $\Pi_{1}^{1}$-norms. Given Polish space $X, Y, \Pi_{1}^{1}$ sets $P \subseteq X, Q \subseteq Y$ and a Borel function $f: X \rightarrow T$ such that $P=$ $f^{-1}[Q]$, and given a norm $\varphi: Q \rightarrow$ ORD, we can define a norm $\psi: P \rightarrow$ ORD by $\psi(x)=\varphi(f(x))$. Then it is easy to verify that if $\varphi$ is a $\Pi_{1}^{1}$-norm on $P, \psi$ is a $\Pi_{1}^{1}$-norm on $Q$.

2. The set of continuous functions with everywhere convergent Fourier series. Let $\mathbf{T}$ denote the unit circle and $C(\mathbf{T})$ the Polish space of continuous real functions on $\mathbf{T}$ with the uniform metric

$$
d(f, g)=\sup \{|f(x)-g(x)|: x \in \mathbf{T}\} .
$$

We can also identify $C(\mathbf{T})$ with the space of all continuous $2 \pi$-periodic real functions on $\mathbf{R}$, viewing $\mathbf{T}$ as $\mathbf{R} / 2 \pi \mathbf{Z}$. Thus we will often view an $f \in C(\mathbf{T})$ as a continuous $f:[0,2 \pi] \rightarrow \mathbf{R}$ with $f(0)=f(2 \pi)$. 
To each $f \in C(\mathbf{T})$ one associates its Fourier series

$$
S[f] \sim \sum_{n=-\infty}^{+\infty} \hat{f}(n) e^{i n t},
$$

where the $n$th Fourier coefficient $\hat{f}(n)$ is given by

$$
\hat{f}(n)=\frac{1}{2 \pi} \int_{0}^{2 \pi} f(t) e^{-i n t} d t .
$$

Let also

$$
S_{N}(f, t)=\sum_{n=-N}^{N} \hat{f}(n) e^{i n t}
$$

be the $N$ th partial sum of the Fourier series of $f$. To say that the Fourier series $S[f]$ converges at a point $t \in[0,2 \pi]$ means that the sequence $\left\{S_{N}(f, t)\right\}$ converges, and in this case we write

$$
S(f, t)=\sum_{n=-\infty}^{+\infty} \hat{f}(n) e^{i n t} .
$$

We will be concerned in this paper with the subset EC of $C(\mathbf{T})$ consisting of all continuous functions with everywhere convergent Fourier series. By a standard theorem (see $[\mathbf{K a}]$ ) if the Fourier series of a continuous function $f$ converges at some point $t$, then it converges to $f(t)$, thus

$$
\begin{aligned}
\mathrm{EC} & =\left\{f \in C(\mathbf{T}): \forall t \in[0,2 \pi]\left(\left\{S_{N}(f, t)\right\} \text { converges }\right)\right\} \\
& =\left\{f \in C(\mathbf{T}): \forall t \in[0,2 \pi]\left(f(t)=\sum_{n=-\infty}^{\infty} \hat{f}(n) e^{i n t}\right)\right\} .
\end{aligned}
$$

In $[\mathbf{K u}]$ Kuratowski gives several applications of the then newly discovered "Kuratowski-Tarski" algorithm, and points out (attributing this to Banach) that EC is a $\Pi_{1}^{1}$ set. He then raises the question of whether this calculation is best possible. We show here that it is, in fact we have

THEOREM. The set EC of continuous functions with everywhere convergent Fourier series is complete $\Pi_{1}^{1}$. In particular it is not Borel.

REMARK. Other examples of coanalytic non-Borel classes of functions are for example: (1) The differentiable functions (see [Maz]), (2) the continuous nowhere differentiable functions (see [Mau] and also [Ke]), (3) the $L^{1}$ functions with everywhere divergent Fourier series (see [Ke]).

One can view the $\Pi_{1}^{1}$-completeness of EC as a consequence of certain results concerning the set of points at which the Fourier series of a continuous function diverges. Let for $f \in C(\mathbf{T})$

$$
D \mathcal{V}_{f}=\left\{x \in[0,2 \pi):\left\{S_{N}(f, x)\right\} \text { diverges }\right\} \text {. }
$$

It is easy to calculate that $D \mathcal{V}_{f}$ is a $G_{\delta \sigma}$ set. Moreover by Carleson's theorem (see $[C]) D V_{f}$ has measure 0 . One now has the following partial converse 
THEOREM (SladKOWSKA [Sl]). Let $B \subseteq(0,2 \pi)$ be an $F_{\sigma}$ set of logarithmic measure 0 and let $A \subseteq B$ be $a G_{\delta \sigma}$. Then there is an $f \in C(\mathbf{T})$ with $D V_{f}=A$.

Recall here that a set $E$ has logarithmic measure 0 if for each $\varepsilon>0$ there is a sequence $\left\{I_{n}\right\}$ of intervals with $E \subseteq \bigcup_{n} I_{n},\left|I_{n}\right|=L_{n}<1$ and $\sum 1 /\left|\log L_{n}\right|<\varepsilon$.

Fix now a perfect set $E \subseteq(0,2 \pi)$ of logarithmic measure 0 . As a particular case of the above theorem we can assign to each $G_{\delta}$ subset $G$ of $E$ an $f(G) \in C(\mathbf{T})$ with $D V_{f(G)}=G$. Then $G=\varnothing \Leftrightarrow D V_{f(G)}=\varnothing \Leftrightarrow f(G) \in$ EC. Now the crucial point is that the construction of $f(G)$ is sufficiently effective, so that given a code $c$ of a $G_{\delta}$ set $G=G_{c}$ one can find in a Borel way the function $f(G)=f\left(G_{c}\right) \equiv F(c)$. Since $P(c) \Leftrightarrow G_{c}=\varnothing \Leftrightarrow$ "c is a $G_{\delta}$ code of the empty set" is a complete $\Pi_{1}^{1}$ set, and $P(c) \Leftrightarrow F(c) \in \mathrm{EC}$, where $F$ is Borel, the same is true for EC. We do not spell out the details of this type of argument (e.g. what we mean by a code for a $G_{\delta}$ etc. ) since we will give shortly a direct proof of the $\Pi_{1}^{1}$-completeness of EC. See however $[\mathbf{K W}, \S 2]$, where an analogous situation is treated in some detail.

Since the exposition in [Sl] is fairly complicated, we present for completeness in an appendix a sketch of a simplified proof of this result, which could be presumably known to the experts in this area. What essentially amounts to a special case of this simpler argument gives a fairly direct and self-contained proof of the $\Pi_{1}^{1}$ completeness of EC, which we give in the next section.

Before we do that however let us say a few more things on the subject of the characterization of the sets $D \mathcal{V}_{f}$. Whether every $G_{\delta \sigma}$ set of measure 0 in $[0,2 \pi)$ can be represented in that form seems to be unknown. Let also for $f \in C(\mathbf{T})$,

$$
\begin{aligned}
\text { UDV } & =\left\{x \in[0,2 \pi):\left\{S_{N}(f, x)\right\} \text { diverges unboundedly }\right\} \\
& =\left\{x \in[0,2 \pi): \varlimsup \lim \left|S_{N}(f, x)\right|=\infty\right\} .
\end{aligned}
$$

Then clearly $U D \mathcal{V}_{f} \subseteq D \mathcal{V}_{f}$ is a $G_{\delta}$ set measure of 0 . The problem has been raised (see for example [Bu1]) whether for every $G_{\delta}$ set $G$ of measure 0 in $[0,2 \pi)$ there is a continuous $f$ with $U D V_{f}=D V_{f}=G$, i.e., $f$ diverges unboundedly on $G$ and converges outside of $G$. It was shown by Buzdalin [Bu2] that this is indeed true if $G$ is both a $G_{\delta}$ and an $F_{\sigma}$ of measure 0 . We sketch in the appendix a simpler argument which actually shows something a bit stronger, namely that the above is true if $G$ is a $G_{\delta}$ contained in an $F_{\sigma}$ set of measure 0 . We do not know if this is new, but we could not locate it in the literature. We will also need a byproduct of this construction in $\S 4$ and we will comment on it there.

3. A proof of the $\Pi_{1}^{1}$-completeness of EC. We give now a direct proof of $\Pi_{1}^{1}$-completeness of EC.

First let us recall that a trigonometric polynomial is any expression of the form

$$
P(t)=\sum_{n=-N}^{N} a_{n} e^{i n t}
$$

with $a_{n} \in \mathrm{C}$. The numbers $-N \leq n \leq N$ with $a_{n} \neq 0$ are called the frequencies of $P$. The trigonometric polynomial $P$ is real if $a_{-n}=\bar{a}_{n}$. We will be dealing with real trigonometric polynomials in the sequel. 
Let for $n \in \mathbf{N}$,

$$
\begin{aligned}
Q(x, n) & =\left[\frac{\cos (n x)}{n}+\frac{\cos (n+1) x}{n-1}+\cdots+\frac{\cos (2 n-1) x}{1}\right] \\
& -\left[\frac{\cos (2 n+1) x}{1}+\frac{\cos (2 n+2) x}{2}+\cdots+\frac{\cos (3 n) x}{n}\right] \\
& \left(=\sum_{m=n}^{2 n-1} \frac{1}{2(2 n-m)}\left(e^{i x m}+e^{-i x m}\right)-\sum_{m=2 n+1}^{3 n} \frac{1}{2(m-2 n)}\left(e^{i x m}+e^{-i x m}\right)\right), \\
& P(x, n)=\frac{\cos (n x)}{n}+\frac{\cos (n+1) x}{n-1}+\cdots+\frac{\cos (2 n-1) x}{1}
\end{aligned}
$$

be the Fejér polynomials. (Thus $P$ is just the "first half" of $Q$.) Two basic properties of the polynomials that we will need are the following:

(1) ( $P$ is large around 0) For $n \in \mathbf{N}$, there is $\delta_{n}>0$ with $|x| \leq \delta_{n} \Rightarrow Q(x, n)>$ $\log n$.

(2) $(Q$ is bounded everywhere) For some absolute constant $C>0$ and all $n \in$ $\mathbf{N}, x \in \mathbf{R}|Q(x, n)|<C$.

The proof of (i) is obvious since $P(0, n)=1+\frac{1}{2}+\cdots+\frac{1}{n}$. For (ii) note that by grouping,

$$
\begin{aligned}
Q(x, n)= & \frac{\cos (n x)-\cos (3 n) x}{n}+\frac{\cos (n+1) x-\cos (3 n-1) x}{n-1} \\
& +\cdots+\frac{\cos (2 n-1) x-\cos (2 n+1) x}{1} \\
= & 2 \sin (2 n x) \cdot \sum_{k=1}^{n} \frac{\sin (k x)}{k} \\
& \therefore|Q(x, n)| \leq 2 \cdot\left|\sum_{k=1}^{n} \frac{\sin (k x)}{k}\right| \leq 2 \cdot(2 \pi+1)
\end{aligned}
$$

(see for example [St, p. 507]).

We will define first a perfect set $E$, homeomorphic to $2^{\mathbf{N}}$. Enumerate $2^{<\mathbf{N}}$ as follows: $\langle()\rangle=0,\langle(0)\rangle=1,\langle(1)\rangle=2,\langle(0,0)\rangle=3,\langle(0,1)\rangle=4,\langle(1,0)\rangle=5$, $\langle(1,1)\rangle=6, \ldots$ Define then inductively on $|s|$, a closed interval $I_{s}$ of $[0,2 \pi]$ with center $x_{s}$ as follows: $I_{()}=[0,2 \pi]$; to define $I_{s^{-}(0)}, I_{s^{-}(1)}$ let first $J_{s}$ be a small enough closed interval with center $x_{s}$, for example having $\left|J_{s}\right| \leq \delta_{2^{\langle s\rangle}{ }^{2}}$ will suffice, and let $I_{s \frown(0)}, I_{s\urcorner(1)}$ be two disjoint subintervals of $J_{s}$.

Finally let

$$
E=\bigcup_{\alpha \in 2^{\mathbf{N}}} \bigcap_{n \in \mathbf{N}} I_{\boldsymbol{\alpha}\lceil n}
$$

The basic lemma about $E$ is now this

LEMMA. Let $0<a<c<d<b<2 \pi$. For each $n, m \in \mathbf{N}$ there is a real trigonometric polynomial $T$ all of whose frequencies have absolute value $>m$ such that

(1) $|T(x)|<1 / 2^{n}$, for all $x$;

(2) The partial sums of $T$ are uniformly bounded by some absolute constant; 
(3) The partial sums $S_{i}(T, x)$ are bounded (in absolute value) by $1 / 2^{n}$ outside $[a, b]$

(4) For some absolute constant $c>0$,

$$
x \in E \cap[c, d] \Rightarrow \max _{i, j}\left|S_{i}(t, x)-S_{j}(t, x)\right| \geq c .
$$

Granting this lemma it is not hard to see that the set WF of wellfounded trees on $\mathbf{N}$ can be reduced via a Borel function to $\mathrm{EC}$, therefore proving the theorem. Indeed define first, inductively on $|u|$, for $u \in \mathbf{N}^{*}$ two open intervals $K_{u} \subseteq L_{u}$ of $(0,2 \pi)$ such that $K_{u} \bigcap E \neq \varnothing$ as follows:

$L_{()}=(0,2 \pi): K_{()}$is some open interval with closure contained in $L_{()}$which intersects $E$ and has length $<\frac{1}{2} \cdot 2 \pi$; to define $L_{u \sim(n)}(n \in \mathbf{N})$ take any disjoint sequence of open intervals $I_{1}, I_{2}, \ldots$, with closures contained in $K_{u}$ each of which intersects $E$, and put $L_{u^{-}(n)}=I_{n}$; let $K_{u^{-} n}$ be some open interval with closure contained in $L_{u^{-}(n)}$, which intersects $E$ and has length $<1 /(|u|+3) \cdot 2 \pi$.

Enumerate now in a 1-1 sequence $u_{1}, u_{2}, \ldots$, the set $\mathbf{N}^{*}$ and let $\left(a_{i}, b_{i}\right)=L_{u_{i}}$, $\left(c_{i}, d_{i}\right)=K_{u_{2}}$ so that $a_{i}<c_{i}<d_{i}<b_{i}$. Let $T_{i}$ be a trigonometric polynomial given by the lemma for $a_{i}<c_{i}<d_{i}<b_{i}$ with $n=i$ and $m$ chosen so that the frequencies of $T_{i}$ have absolute values bigger than those of $T_{j}, j<i$.

Given now a tree $T$ on $\mathbf{N}$ associate to it the function $f_{T}=\Sigma\left\{T_{i}: u_{i} \in T\right\}=$ $\sum_{u_{i} \in T} T_{i}$. Since $\left|T_{i}(x)\right|$ is bounded everywhere by $1 / 2^{i}, f_{T} \in C(\mathbf{T})$. We will show that

$$
T \in \mathrm{WF} \Leftrightarrow f_{T} \in \mathrm{EC} .
$$

Since the map $T \mapsto f_{T}$ is clearly Borel this will complete the proof.

Let $P(T)=\left\{i \in \mathbf{N}: u_{i} \in T\right\}$, so that $f_{T}=\sum_{i \in P(T)} T_{i}$. The basic observation is that if $T \in \mathrm{WF}$ then for every $x \in[0,2 \pi], x$ belongs only to finitely many intervals $\left[a_{i}, b_{i}\right], i \in P(T)$, while if $T \notin \mathrm{WF}$ there is some $x \in E$ such that $x$ belongs to infinitely many intervals $\left[c_{i}, d_{i}\right]$. Thus it is enough to show that

(a) $x$ belongs to only finitely many $\left[a_{i} . b_{i}\right], i \in P(T) \Rightarrow\left\{S_{N}\left(f_{T}, x\right)\right\}$ converges,

(b) $x \in E$ belongs to infinitely many $\left[c_{i}, d_{i}\right], i \in P(T) \Rightarrow\left\{S_{N}\left(f_{T}, x\right)\right\}$ diverges.

First notice that because of the uniform convergence of $\sum_{i \in P(T)} T_{i}$ and the noninterference of the frequencies of the $T_{i}$ 's the Fourier series of $f_{T}$ is exactly this sum (after "removing the parentheses around each $T_{i}$ ").

ProOF OF (a). Let $i_{0}$ be such that for $i \in P(T), i \geq i_{0}, x \notin\left[a_{i}, b_{i}\right]$. Fix $\varepsilon>0$. Then for $i \in P(T), i \geq i_{0}$,

$$
S_{n}\left(f_{T}, x\right)=S_{n}\left(\sum_{\substack{j<i \\ j \in P(T)}} T_{j}, x\right)+\sum_{\substack{j \geq i \\ j \in P(T)}} S_{n}\left(T_{j}, x\right) .
$$

Choose $i_{1}(\varepsilon) \equiv i_{1}>i_{0}$ with $\sum_{\jmath \geq i_{1}} 1 / 2^{\jmath}<\varepsilon$. Then choose $n_{0}(\varepsilon) \equiv n_{0}$ bigger than all the absolute values of the frequencies of $T_{\jmath}, j<i_{1}$. Then

$$
\left|S_{n+p}\left(\sum_{\substack{j<i_{1} \\ j \in P(T)}} T_{j}, x\right)-S_{n}\left(\sum_{\substack{j<2_{1} \\ \jmath \in P(T)}} T_{\jmath}, x\right)\right|=0,
$$


for any $p>0, n \geq n_{0}$. So for $p>0, n \geq n_{0}$

$$
\left|S_{n+p}\left(f_{T}, x\right)-S_{n}\left(f_{T}, x\right)\right| \leq\left|\sum_{\substack{j \geq i_{1} \\ j \in P(T)}} S_{n+p}\left(T_{j}, x\right)\right|+\left|\sum_{\substack{j \geq i_{1} \\ j \in P(T)}} S_{n}\left(T_{j}, x\right)\right| .
$$

But since $x \notin\left[a_{j}, b_{j}\right]$, for $j \geq i_{1}, j \in P(T)$ we have that $\left|S_{k}\left(T_{j}, x\right)\right| \leq 1 / 2^{j}$ for such $j$ 's by (3) of the lemma, thus the above sum is bounded by

$$
2 \cdot \sum_{j \geq i_{1}} \frac{1}{2^{j}} \leq 2 \varepsilon
$$

and thus $\left|S_{n+p}\left(f_{T}, x\right)-S_{n}\left(f_{T}, x\right)\right| \leq 2 \varepsilon$, for all $n \geq n_{0}(\varepsilon), p>0$, so that $\left\{S_{n}\left(f_{T}, x\right)\right\}$ converges.

PROOF OF (b). By (4) of the lemma and since $x \in E \cap\left[c_{i}, d_{i}\right]$ for infinitely many $i \in P(T)$, we have infinitely many $j_{i}<j_{2}$ with $\left|S_{j_{1}}\left(f_{T}, x\right)-S_{j_{2}}\left(f_{T}, x\right)\right| \geq c>0$, so that $\left\{S_{N}\left(f_{T}, x\right)\right\}$ diverges.

It remains only to give the

PROOF OF THE LEMMA. Let

$$
f(x)=\sum_{k=1}^{\infty} \frac{1}{k^{2}} Q\left(x-x_{k}, 2^{k^{2}}\right),
$$

where $x_{k}=x_{s}$ for $\langle s\rangle=k$. This is a continuous function and the expression on the right is its Fourier series (after "removing parentheses" - note again that there is no interference in the frequencies of two different summands). Let $\lambda(x)$ be a $C^{\infty} 2 \pi$-periodic function which is $\equiv 1$ on $[c, d]$ and $\equiv 0$ outside $[a, b]$, and has absolute value $\leq 1$ everywhere. Then by a standard theorem (see [Zy, I, p. 53]) $\lim _{N \rightarrow \infty}\left(S_{N}(\lambda f, x)-\lambda(x) S_{N}(f, x)\right)=0$ uniformly on $x \in[0,2 \pi]$, so choose $m_{0}>m$ such that for $N \geq m_{0}$ and all $x$

$$
\left|S_{N}(\lambda f, x)-\lambda(x) S_{N}(f, x)\right|<\frac{1}{4} \cdot \frac{1}{2^{n}} .
$$

Choose now $m_{1}>m_{0}$ such that

$$
C \cdot\left(\sum_{m=m_{1}}^{\infty} \frac{1}{\dot{m}^{2}}\right)<\frac{1}{2} \cdot \frac{1}{2^{n}},
$$

where $C$ is the constant given in property (2) of the Fejér polynomials.

Note now that from our enumeration of $2^{<N}$ the binary sequences of length $l \geq 1$ have exactly the code numbers between

$$
p(l)=\langle(\underbrace{0, \ldots, 0}_{l})\rangle=2^{l}-1
$$

and

$$
q(l)=(\langle\underbrace{1, \ldots, 1}_{l}\rangle)=2^{l+1}-2 .
$$

Also the frequencies of $Q\left(x-x_{k}, 2^{k^{2}}\right)$ are (in absolute value) strictly between $\mu(k)=$ $2^{k^{2}}-1$ and $\nu(k)=3 \cdot 2^{k^{2}}+1$. Finally let $l=m_{1}+1$ and put

$$
T(x)=S_{\nu(q(l))}(\lambda f, x)-S_{\mu(p(l))}(\lambda f, x) .
$$


By $(*)$ this is a close approximation of $\lambda(x)$ multiplied by

$$
\begin{aligned}
\tilde{T}(x) & =S_{\nu(q(\lambda))}(f, x)-S_{\mu(p(l))}(f, x) \\
& =\sum_{|s|=l} \frac{1}{\langle s\rangle^{2}} Q\left(x-x_{s}, 2^{\langle s\rangle^{2}}\right) .
\end{aligned}
$$

Clearly the frequencies of $T(x)$ have absolute value $>m$. We verify now (1)-(4) of the lemma.

(1) By $(*)$ it is enough to check that $|\tilde{T}(x)|<(1 / 2) \cdot\left(1 / 2^{n}\right)$ for all $x$. But

$$
|\tilde{T}(x)|<\left(\sum_{m=m_{1}}^{\infty} \frac{1}{m^{2}}\right) \cdot C<\frac{1}{2} \cdot \frac{1}{2^{n}} .
$$

(2) Note that every partial sum $S_{N}(\lambda f, x)$ of $\lambda f$ for $N>m_{1}$ is bounded by $1+\left|S_{N}(f, x)\right|$, so it is enough to show these partial sums $S_{N}(f, x)$ of $f$ are bounded. But any partial sum of $f$ has the form $\sum_{k=1}^{p-1} k^{-2} Q\left(x-x_{k}, 2^{k^{2}}\right)+$ a partial sum of $\left(1 / p^{2}\right) Q\left(x-x_{p}, 2^{p^{2}}\right)$. From the definition of $Q$ it is easy to verify that any partial sum of $Q(x, n)$ is bounded by $2\left(1+\frac{1}{2}+\cdots+\frac{1}{n}\right)$ therefore by $4 \log n$. So every partial sum of $p^{-2} Q\left(x-x_{p}, 2^{p^{2}}\right)$ is bounded by $1 / p^{2} \cdot 4 \cdot \log \left(2^{p^{2}}\right)=4 \log 2$ and we are done.

(3) Note that a partial sum of $T$ is a difference of two partial sums $S_{i}(\lambda f, x)$ and $S_{j}(\lambda f, x)$ of $\lambda f$, for $\mu(p(l)) \leq i, j \leq \nu(q(l))$. So it is enough to show that for $\mu(p(l)) \leq-i \leq \nu(q(\lambda)),\left|S_{i}(\lambda f, x)\right| \leq 2 \cdot 1 / 2^{n+1}$ for $x \notin[a, b]$. But $\lambda(x)=0$ for $x \notin[a, b]$ so we are done by $(*)$.

(4) Let now $x \in E \cap[c, d]$. Let $\alpha \in 2^{\mathbf{N}}$ be such that $x \in \bigcap_{n} I_{\alpha \uparrow n}=\bigcap_{n} J_{\alpha \mid n}$. In particular $x \in J_{\alpha \uparrow l}$. Say $s=\alpha \uparrow l$ and $\langle s\rangle=k$. Thus $p(l) \leq k \leq q(l)$ and $\mu(p(l)) \leq \mu(k) \leq \nu(k) \leq \nu(q(l))$. Note that $\langle s\rangle^{-2} P\left(x-x_{s}, 2^{\langle s\rangle^{2}}\right)$ is the difference between two partial sums of $f$, say $S_{i}(f, x)-S_{j}(f, x)$ where $i=\mu(k), j<\nu(k)$. Since $x \in J_{s}$ and $\left|J_{s}\right|<\delta_{2^{\langle s\rangle^{2}}}$ we have $\left|x-x_{s}\right| \leq \delta_{2^{\langle s\rangle^{2}}}$, thus $P\left(x-x_{s}, 2^{\langle s\rangle^{2}}\right)>$ $\log \left(2^{\langle s\rangle^{2}}\right)=\langle s\rangle^{2} \cdot \log 2$, so $S_{i}(f, x)-S_{j}(f, x)>\log 2$. The rest is really obvious: we have (remembering that $\lambda(x)=1$, since $x \in[c, d]$ )

$$
\begin{aligned}
& \left|S_{j}(T, x)-S_{i}(T, x)\right|=\left|S_{j}(\lambda f, x)-S_{i}(\lambda f, x)\right| \\
& \quad \geq\left|S_{j}(f, x)-S_{i}(f, x)\right|-1 / 2 \cdot 2^{n} \\
& \quad>\log 2-1 / 2 \cdot 2^{n}>\log 2-\frac{1}{2}=c>0 .
\end{aligned}
$$

REMARK. Note that we have not used property (2) of the lemma in the proof of the $\Pi_{1}^{1}$-completeness of EC. We can use it however to obtain a stronger result. Let

$$
\mathrm{EC}_{1}=\{f \in C(\mathbf{T}): f \text { has partial sums bounded }
$$

(in absolute value) by 1 and $f \in \mathrm{EC}\}$.

Then we have

THEOREM. The set $\mathrm{EC}_{1}$ is $\Pi_{1}^{1}$-complete. In fact there is a Borel function $f: 2^{\mathbf{N}^{*}} \rightarrow C(\mathbf{T})$ such that $T \in \mathrm{WF} \Rightarrow f(T) \in \mathrm{EC}_{1}$ and $T \notin \mathrm{WF} \Rightarrow f(T) \notin \mathrm{EC}$.

In particular this implies that there is no Borel set $B$ with $\mathrm{EC}_{1} \subseteq B \subseteq \mathrm{EC}$. We will use this in the next section. 
4. The Zalcwasser rank. We will introduce now a natural $\Pi_{1}^{1}$-norm on EC. The ordinal rank attached to each $f \in$ EC will measure how "nicely" the Fourier series of $f$ converges.

Let $a<b$ be real numbers and let $C[a, b]$ be the space of continuous functions on $[a, b]$ with the uniform metric. Consider then the Polish space $X=C[a, b]^{\mathbf{N}}$ of all infinite sequences $\left\{f_{n}\right\}$ from $C[a, b]$. Let

$$
C N=\left\{\left\{f_{n}\right\} \in X: \forall x \in[a, b]\left(\left\{f_{n}(x)\right\} \text { converges }\right)\right\}
$$

Then $C N$ is a complete $\Pi_{1}^{1}$ subset of $X$. Zalcwasser [Za] and independently Gillespie and Hurewicz $[\mathbf{G H}]$ have assigned to each $\left\{f_{n}\right\} \in C N$ a countable ordinal number in order to prove by transfinite induction that for every $\left\{f_{n}\right\} \in C N$ with $\left\{\left\|f_{n}\right\|_{\infty}\right\}$ bounded and $\lim f_{n}=f$ continuous, there is a sequence of functions $\left\{\varphi_{n}\right\}$ in $C[a, b]$ each of which is a convex combination of functions in $f_{n}$ (i.e. has the form $\left.\lambda_{1} \cdot f_{n_{1}}+\cdots+\lambda_{k} \cdot f_{n_{k}}, \lambda_{i} \geq 0, \sum_{i=1}^{k} \lambda_{i}=1\right)$ such that $\varphi_{n} \mapsto f$ uniformly. This is of course a very special case of Mazur's theorem that in a metrizable locally convex space $B$, if $\left\{x_{n}\right\}$ is a sequence in $X$ which converges weakly to $x \in B$ then there is a sequence $\left\{y_{i}\right\}$ of convex combinations of $\left\{x_{n}\right\}$ which converges to $x$ (in the topology of $B$ ). (See e.g. [R, 3.13].) Use of ordinal indices in the theory of Banach spaces in Szlenk [Sz] (and subsequent papers) seems also to have been motivated by the construction of Zalcwasser and Gillespie-Hurewicz.

We will explain now the definition and basic properties of this rank function on $C N$ and we will then show that it defines a $\Pi_{1}^{1}$-norm on $C N$. By specializing it to the Fourier series of a continuous function we will then obtain the $\Pi_{1}^{1}$-norm on EC.

DEFINITION. Let $\bar{f}=\left\{f_{n}\right\}$ be a sequence of continuous functions on $[a, b]$. Let $P \subseteq[a, b]$ be a closed set and let $x \in P$. We define the value of the oscillation function of $\bar{f}$ on $P$ at $x$ as follows

$$
\omega_{\bar{f}}(x, P)=\inf _{\delta>0} \inf _{p \in \mathbf{N}} \sup \left\{\left|f_{m}\left(x^{\prime}\right)-f_{n}\left(x^{\prime}\right)\right|: m>n \geq p \& x^{\prime} \in P \&\left|x^{\prime}-x\right|<\delta\right\} .
$$

Definition. For each $\bar{f}=\left\{f_{n}\right\} \in C[a, b]^{\mathbf{N}}$ and each $\varepsilon \in \mathbf{Q}^{+}$(= the set of positive rationals) define a "derivative operation" on closed subsets of $[a, b]$ as follows: Given a closed set $P \subseteq[a, b]$ let

$$
P_{\varepsilon, \bar{f}}^{\prime} \equiv P^{\prime}=\left\{x \in P: \omega_{\bar{f}}(x, P) \geq \varepsilon\right\}
$$

Clearly $P_{\varepsilon}^{\prime}$ is a closed subset of $P$. Thus we can define inductively for each $\bar{f} \in$ $C[a, b]^{\mathbf{N}}, \varepsilon \in \mathbf{Q}^{+}$, a closed set $P_{\varepsilon, f}^{\alpha} \equiv P_{\varepsilon}^{\alpha}$, for $\alpha$ an ordinal, as follows:

$$
\begin{aligned}
& P_{\varepsilon}^{0}=[a, b], \quad P_{\varepsilon}^{\alpha+1}=\left(P_{\varepsilon}^{\alpha}\right)^{\prime}, \\
& P_{\varepsilon}^{\lambda}=\bigcap_{\alpha<\lambda} P_{\varepsilon}^{\alpha}, \quad \text { for } \lambda \text { limit. }
\end{aligned}
$$

Then $\alpha \leq \beta \Rightarrow P_{\varepsilon}^{\alpha} \leq P_{\varepsilon}^{\beta}$ (and note also that $\varepsilon \leq \varepsilon^{\prime} \Rightarrow P_{\varepsilon^{\prime}}^{\alpha} \subseteq P_{\varepsilon}^{\alpha}$ ). So for each $\varepsilon \in \mathbf{Q}^{+}$there is a least $\alpha_{\bar{f}}(\varepsilon) \equiv \alpha(\varepsilon)<\omega_{1}$ such that $P_{\varepsilon}^{\alpha}=P_{\varepsilon}^{\alpha(\varepsilon)} \equiv P^{\infty}$ for all $\alpha \geq \alpha(\varepsilon)$. We have now the following

FACT 4.1 (ZalCWASSER, Gillespie-HuREWiCZ). Let $\bar{f} \in C[a, b]^{\mathbf{N}}$. Then

$$
\begin{aligned}
\bar{f} \in C N & \Leftrightarrow \forall \varepsilon \in \mathbf{Q}^{+}\left(P_{\varepsilon}^{\infty}=\varnothing\right) \\
& \Leftrightarrow \forall \varepsilon \in \mathbf{Q}^{+} \exists \alpha<\omega_{1}\left(P_{\varepsilon}^{\alpha}=\varnothing\right) \\
& \left(\Leftrightarrow \forall n \exists \alpha<\omega_{1}\left(P_{1 / n}^{\alpha}=\varnothing\right)\right) .
\end{aligned}
$$


PROOF. $(\Rightarrow)$ : It is enough to show the following

LEMMA 4.1.1. Let $f \in C[a, b]^{\mathbf{N}}$ and assume $\varnothing \neq P$ is closed and $\forall x \in P$ $\left\{f_{n}(x)\right\}$ converges. Then $P_{\varepsilon}^{\prime}$ is nowhere dense in $P$, and thus $P_{\varepsilon}^{\prime} \varsubsetneqq P$, for all $\varepsilon \in \mathbf{Q}^{+}$.

PROOF. Assume that for some $\varepsilon \in \mathbf{Q}^{+}$, there is an open interval $I$ with $\varnothing \neq$ $I \cap P \subseteq P_{\varepsilon}^{\prime}$. Set

$$
G_{p}=\left\{x \in P: \exists m>n \geq p\left|f_{m}(x)-f_{n}(x)\right|>\varepsilon / 2\right\} .
$$

Then $G_{p}$ is open and dense in $I \cap P$. This is because if $\varnothing \neq I^{\prime} \cap P \subseteq I \cap P, I^{\prime}$ an open interval, let $x \in I^{\prime} \cap P$. Let also $\delta>0$ be so small that $\left|x^{\prime}-x\right|<\delta \Rightarrow x^{\prime} \in I^{\prime}$. Since $x \in P_{\varepsilon}^{\prime}$ we have that

$$
\sup \left\{\left|f_{m}\left(x^{\prime}\right)-f_{n}\left(x^{\prime}\right)\right|: m>n \geq p \& x^{\prime} \in P \&\left|x^{\prime}-x\right|<\delta\right\}>\varepsilon / 2,
$$

thus find $x^{\prime} \in P,\left|x^{\prime}-x\right|<\delta$ with $\left|f_{m}\left(x^{\prime}\right)-f_{n}\left(x^{\prime}\right)\right|>\varepsilon / 2$ for some $m>n \geq p$. Then clearly $x^{\prime} \in G_{p}$, and $x^{\prime} \in I^{\prime} \cap P$.

By the Baire category theorem $\bigcap_{p} G_{p} \neq \varnothing$. If $x_{0} \in \bigcap_{p} G_{p}$ then clearly $\left\{f_{n}\left(x_{0}\right)\right\}$ diverges, a contradiction.

$(\Leftarrow)$ : Assume that $\left\{f_{n}\right\} \notin C N$. Let $x \in[a, b]$ be such that $\left\{f_{n}(x)\right\}$ diverges. Choose $\varepsilon>0$ such that for all $p$ there is $n>m \geq p$ with $\left|f_{n}(x)-f_{m}(x)\right| \geq \varepsilon$. Then clearly (by induction on $\alpha$ ), $x \in P_{\varepsilon}^{\alpha}$ for all $\alpha$, thus $P_{\varepsilon}^{\infty} \neq \varnothing$.

Thus if $\bar{f} \in C N$, there is for each $\varepsilon \in \mathbf{Q}^{+}$, a least countable ordinal $\alpha(\varepsilon)$ such that $P_{\varepsilon}^{\alpha(\varepsilon)}=\varnothing$. (Obviously $\alpha(\varepsilon)$ is a successor ordinal.) Thus we can define a rank function for $C N$ as follows:

DEFINITION. For each $\bar{f} \in C N$ let

$$
\begin{aligned}
|\bar{f}| & =\sup \left\{\alpha(\varepsilon): \varepsilon \in \mathbf{Q}^{+}\right\} \quad(=\sup \{\alpha(1 / n): n \in \mathbf{N}\}) \\
& =\text { the least ordinal } \alpha \text { for which } P_{\varepsilon}^{\alpha}=\varnothing, \text { for all } \varepsilon \in \mathbf{Q}^{+} .
\end{aligned}
$$

Clearly the smallest possible rank is 1 . Sequences with this least possible rank should be "nicely" convergent. This is indeed the case.

FACT 4.2 (ZALCWASSER, GILLESPIE-HUREWICZ). Let $\bar{f}=\left\{f_{n}\right\} \in C N$. Then $|\bar{f}|=1 \Leftrightarrow\left\{f_{n}\right\}$ is uniformly convergent.

PROOF. Assume $\left\{f_{n}\right\}$ converges uniformly. Then for each $\varepsilon>0$ there is $p$ so that if $n>m \geq p$, we have $\left|f_{n}(x)-f_{m}(x)\right|<\varepsilon$, all $x$. Thus clearly $\omega_{\bar{f}}(x,[a, b])=0$, so $P_{\varepsilon}^{1}=\varnothing$ and $|\bar{f}|=1$.

Assume conversely that $|\bar{f}|=1$. Then for any $x \in[a, b], \omega_{\bar{f}}(x,[a, b])=0$. Fix $\varepsilon>0$. Let $x \in[a, b]$ and find $\delta_{x}>0, p_{x} \in \mathbf{N}$ such that

$$
\sup \left\{\left|f_{m}\left(x^{\prime}\right)-f_{n}\left(x^{\prime}\right)\right|: m>n \geq p_{x} \&\left|x^{\prime}-x\right|<\delta_{x}\right\}<\varepsilon .
$$

By compactness find $x_{1}, \ldots, x_{n} \in[a, b]$ such that if $x \in[a, b]$ then for some $1 \leq i \leq$ $n,\left|x-x_{i}\right|<\delta_{x_{i}}$. Let $p=\max _{1 \leq i \leq n} p_{x_{i}}$. Then for any $x$

$$
\sup _{m>n \geq p}\left|f_{m}(x)-f_{n}(x)\right| \leq \sup _{m>n \geq p_{x_{2}}}\left|f_{m}(x)-f_{n}(x)\right|<\varepsilon
$$

where $\left|x-x_{i}\right|<\delta_{x_{i}}$. Thus $\left\{f_{n}\right\}$ converges uniformly.

The basic definability property of the rank function $\bar{f} \mapsto|\bar{f}|$ is expressed in the following fact. 
FACT 4.3. The rank function $\bar{f} \mapsto|\bar{f}|$ is a $\Pi_{1}^{1}$-norm on $C N$.

The proof is entirely similar to that of Fact 3.6. in [KW] and we omit it here.

By specializing to the partial sums of the Fourier series of a continuous function we obtain a $\Pi_{1}^{1}$-norm on EC.

DEFINITION. Let $f \in$ EC. The Zalcwasser rank of $f$ is the ordinal $|f|_{Z}=$ $\left|\left\{S_{n}(f)\right\}\right|$, where $S_{n}(f)(x)=S_{n}(f, x)$.

Since the map $f \mapsto\left\{S_{n}(f)\right\}$ is clearly a Borel map from $C(\mathbf{T})$ into $C[0,2 \pi]^{\mathbf{N}}$ and $f \in \mathrm{EC} \Leftrightarrow\left\{S_{n}(f)\right\} \in C N$, it follows immediately that we have the following

FACT 4.4. The Zalcwasser rank is a $\Pi_{1}^{1}$-norm on $E C$.

From 4.2. we also have

FACT 4.5 . Let $f \in$ EC. Then

$$
|f|_{z}=1 \Leftrightarrow \text { the Fourier series of } f \text { converges uniformly. }
$$

There are some standard examples of continuous functions whose Fourier series converge everywhere but not uniformly. For instance see [Ba, I, p. 125] for Fejér's example of an $F_{0} \in$ EC whose Fourier series converges uniformly on any closed interval avoiding 0 but not on $[0,2 \pi]$. It follows that $\left|F_{0}\right|_{z}=2$, so this is an example of such a behavior of least complexity. By the method of "condensation of singularities" one can easily produce an example of an $F_{1} \in \mathrm{EC}$ such that the Fourier series of $F_{1}$ converges nonuniformly in any interval. Indeed let $r_{1}, r_{2}, \ldots$, be the rationals in $[0,2 \pi]$. Let then $F_{1}(x)=\sum_{k=1}^{\infty} 2^{-k} F_{0}\left(x-r_{k}\right) ; F_{1}$ easily works (see [Ba, I, p. 342]). Again however $\left|F_{1}\right|_{z}=2$. This is because for each $\varepsilon>0 P_{\varepsilon}^{1}$ $\left(\equiv P_{\varepsilon,\left\{S_{n}\left(F_{1}\right)\right\}}^{1}\right)$ consists of a finite set of rationals, thus $P_{\varepsilon}^{2}=\varnothing$.

One can see however that there must exist functions in EC whose Fourier series have arbitrarily large Zalcwasser rank, i.e. exhibit arbitrarily complicated convergence behavior.

FACT 4.6. For each ordinal $\alpha<\omega_{1}$ there is a continuous function $f$ whose Fourier series converges everywhere and has partial sums bounded by 1 , such that $|f|_{z}>\alpha$.

PROOF. We have seen at the end of $\S 3$ that there is no Borel set between $\mathrm{EC}_{1}$ and EC. Since $f \mapsto|f|_{z}$ is a $\Pi_{1}^{1}$-norm on EC, $\left\{f \in \mathrm{EC}:|f|_{z} \leq \alpha\right\}$ is a Borel subset of $\mathrm{EC}$, so some $f \in \mathrm{EC}_{1}$ avoids it, i.e. there is $f \in \mathrm{EC}_{1}|f|_{z}>\alpha$.

One can obtain further results here. Recall the standard fact (see e.g. [Ba, I, p. 114]) that every differentiable function $f \in C(\mathbf{T})$ has everywhere convergent Fourier series. So one can ask whether the convergence behavior of the Fourier series of a differentiable function can be also arbitrarily complicated. This is indeed the case. Letting $D=\{f \in C(\mathbf{T}): f$ is differentiable $\}$, so that $D \subseteq \mathrm{EC}$, it will follow from Theorem A.2 in the Appendix that no Borel set $B$ exists with $D \subseteq B \subseteq$ EC. Thus as before

FACT 4.7. For each ordinal $\alpha<\omega_{1}$ there is a differentiable function $f$ such that $|f|_{z}>\alpha$.

T. Ramsamujh has verified that if $f$ is a function of bounded variation on $\Pi$, then $\left|\left\{S_{n}(F)\right\}\right| \leq 2$. This points out an interesting qualitative difference in the convergence behavior of the Fourier series of differentiable functions vs. those of bounded variation.

It follows also from Fact 4.7 that the set of Fourier series of differentiable functions is not a Borel set (in $c_{0}(\mathbf{Z})$ ). Thus there can be no "reasonable" criteria 
for characterizing when a given Fourier series $\Sigma a_{n} e^{i n x}$ is that of a differentiable function. (Again there are well-known Borel criteria (see e.g. [Ba]) for the case of functions of bounded variation.)

\section{Remarks and problems.}

5.1. In $\S 4$ we have shown that there are functions in EC of arbitrarily large Zalcwasser rank. It would be interesting to construct explicitly for each ordinal $\alpha$ a function in EC of rank exceeding $\alpha$. This would give in particular a different proof of the non-Borelness of EC.

5.2. In $\S 4$ we have seen that each of $D$ and $\mathrm{EC}_{1}$ is unbounded in the Zalcwasser norm. Is this true for $D \cap \mathrm{EC}_{1}$, i.e. can one construct functions of arbitrarily large Zalcwasser rank which are both differentiable and whose Fourier series have bounded partial sums?

5.3. It is a standard result, as we mentioned earlier, that $D \subseteq$ EC. To each $f \in D$ one associates (see $[\mathbf{K W}]$ ) an ordinal rank $|f| \equiv|f|_{D}$ which measures the "niceness" of the derivative of $f$. Thus one would like to find a quantitative version of the inclusion $D \subseteq$ EC which would relate $|f|_{D}$ and $|f|_{Z}$, when $f \in D$. If $|f|_{D}=1$, then $f^{\prime}$ is continuous, so clearly $|f|_{Z}=1$ (since the Fourier series of $f$ converges absolutely, therefore uniformly). So it is natural to guess that always $f \in D \Rightarrow|f|_{D} \geq|f|_{Z}$.

To each $f \in D$ one can also associate another ordinal, called the Denjoy rank of $f$, say $|f|_{D J}$, which measures the number of steps it takes to recover $f$ from $f^{\prime}$ via the Denjoy process (see $[\mathbf{B r}]$ ). T. Ramsamujh has shown that $|f|_{D} \geq|f|_{D J}$. Now $|f|_{D J}=1$ iff $f^{\prime}$ is integrable. So again if $|f|_{D J}=1$ we have that the Fourier series of $f$ is uniformly convergent, so $|f|_{Z}=1$. Thus it is reasonable to propose the following stronger conjecture:

$$
f \in D \Rightarrow\left[|f|_{D J} \geq|f|_{Z}\right]
$$

Appendix. We sketch first a simpler proof, using ideas from [EHP], [Ze] (see also [Ba, I, pp. 470-479]) of

THEOREM A1 (SLADKOWSKA [Sl]). Let $A \in G_{\delta \sigma}$ and assume there is $B \subseteq$ $[0,2 \pi), B \in F_{\sigma}$, of logarithmic measure 0 with $A \subseteq B$. Then there is a continuous function $f \in C(\mathbf{T})$ with $\|f\|_{\infty} \leq 1$ and $\left\|S_{N}(f)\right\|_{\infty} \leq 1$ for all $N$, and such that the Fourier series of $f$ diverges exactly at the points in $A$ (modulo $2 \pi$ ).

ProOF. First notice that it is enough to handle the special case when $A \in G_{\delta}$ and $B$ is closed. So assume $B=F$ is closed and $A=E$ is a $G_{\delta}$. Since $E \in G_{\delta}$ there is a sequence $0 \leq a_{i}<c_{i}<b_{i} \leq 2 \pi$ such that every $x \in E$ belongs to infinitely many $\left[c_{i}, d_{i}\right]$, but every $x \notin E$ belongs only to finitely many $\left[a_{i}, b_{i}\right]$. The result then follows easily from the following.

LEMMA 1. Let $0 \leq a<b<c<d \leq 2 \pi$. For $m, n$ there is a real trigonometric polynomial $T_{m, n}=T$ such that (1) $\|T\|_{\infty} \leq 1 / 2^{n},(2)\left\|S_{k}(T)\right\|_{\infty} \leq 1$, for all $k,(3)$ for some absolute constant $\rho>0$, if $x \in F \cap[c, d]$, then $\max _{p, q}\left|S_{p}(T, x)-S_{q}(T, x)\right| \geq$ $\rho$, (4) if $x \notin[a, b]$, then $\left|S_{k}(t, x)\right| \leq 1 / 2^{n}$ for all $k$, and (5) the frequencies of $T$ have absolute values $>m$.

Granting this lemma define inductively trigonometric polynomials $T_{i}$ for $a_{i}, c_{i}$, $d_{i}, b_{i}, n=i$ and $m>$ the absolute values of the frequencies of $T_{j}, j<i$. Let finally $f=\Sigma_{i} T_{i}$. This is easily seen to work as in $\S 3$. 
It remains to prove the lemma. For that one needs a construction of real trigonometric polynomials with certain behavior on a given interval due to Erdös, Herzog and Piranian [EHP].

LEMMA 2. Let $I \subseteq[0,2 \pi]$ be an interval of length $L<\frac{1}{4}$. For each $m$ there is a trigonometric polynomial $Q_{m}=Q$ such that (1) $\|Q\|_{\infty} \leq 1 /|\log L|,(2)$ $\left\|S_{k}(Q)\right\|_{\infty} \leq 1$, (3) for some absolute constant $\rho_{1}>0$,

$$
\max _{p, q}\left|S_{p}(Q, x)-S_{q}(Q, x)\right| \geq \rho_{1} \quad \text { for all } x \in I
$$

and (4) the frequencies of $Q$ have absolute values $>m$.

From that the proof of Lemma 1 is completed as follows: Let $F \subseteq \bigcup_{n} I_{n}$ where $\left|I_{n}\right|=L_{n}<\frac{1}{4}, \Sigma 1 /\left|\log L_{n}\right|<1$ and every $x \in F$ belongs to infinitely many $I_{n}$ 's. By Lemma 2 there are trigonometric polynomials $Q_{n}$ having the above properties for $I_{n}$ and with frequencies of absolute value between $\mu_{n}, \nu_{n}$ where $\mu_{n}<\nu_{n}<$ $\mu_{n+1}<\nu_{n+1}$. Put $f=\Sigma Q_{n}$. Let $\lambda \in C(\mathbf{T})$ be in $C^{\infty}, \lambda \equiv 1$ on $[c, d], \lambda \equiv 0$ off $[a, b],\|\lambda\|_{\infty} \leq 1$. Then $S_{i}(\lambda f, x)-\lambda(x) S_{i}(f, x) \rightarrow 0$ uniformly. So choose $n_{0}>m$ so that $\left|S_{i}(\lambda f, x)-\lambda(x) S_{i}(f, x)\right|<\left(1 / 4 \cdot 2^{n}\right) \cdot \rho_{1}$ for $i>n_{0}$ and all $x$. Choose $n_{1}>n_{0}$ so that $\sum_{i=n_{1}}^{\infty} 1 /\left|\log L_{i}\right|<1 / 2^{n+1}$. Then for each $x \in F \cap[c, d]$ let $i_{x}>n_{1}$ be such that $\max _{\mu_{n_{1}}<p, q<\nu_{\imath_{x}}}\left|S_{p}(f, x)-S_{q}(f, x)\right|>\rho_{1}$. By compactness there is $i_{1}>n_{1}$ with

$$
x \in F \cap[c, d] \Rightarrow \max _{\mu_{n_{1}}<p, q<\nu_{i_{1}}}\left|S_{p}(f, x)-S_{q}(f, x)\right|>1 / 2 .
$$

Put finally $\tilde{T}(x)=S_{\nu_{i_{1}}(\lambda f, x)}-S_{\mu_{n_{1}}(\lambda f, x)}$. Then $T=\tilde{T} / 6$ easily works as in $\S 3$.

We finally outline the proof of a strengthening of the result in [Bu2], using ideas from [Ka and Ze]. We state it in a form that is needed for the conclusion concerning the Zalcwasser rank of differentiable functions in $\S 4$.

THEOREM A2. Let $E \in G_{\delta}, F \in F_{\sigma}, E \subseteq F, F \subseteq[0,2 \pi)$ and assume $F$ has measure 0 . Then there is a continuous function $f \in C(\mathbf{T})$ such that the Fourier series of $f$ diverges unboundedly on $E$ and $f^{\prime}(x)$ exists for every $x \notin E$ (in particular the Fourier series of $f$ converges off $E$ ).

ProOF. Again we need the following

LEMMA. Let $H$ be a closed set of measure 0 . Let $0 \leq a<c<d<b \leq 2 \pi$. For each $\varepsilon>0, m$ there is a real trigonometric polynomial $T_{\varepsilon, m}=T$ such that (1) $|T|_{\infty} \leq \varepsilon,(2)$ if $x \in H \cap[c, d]$, then $\max _{p, q}\left|S_{p}(t, x)-S_{q}(T, x)\right|>1 / \varepsilon,(3)$ if $x \notin[a, b]$, then $\left|S_{k}(T, x)\right| \leq \varepsilon$ for all $k$, (4) if $x \notin[a, b]$, then $|(T(x+h)-T(x)) / h| \leq \varepsilon$, for all $h$ and (5) the frequencies of $T$ have absolute values $>m$.

Granting this lemma the proof can be completed as before. For the proof of the lemma notice that by [Ka and Bu1] there is a real function $g \in C(\mathbf{T})$ such that the Fourier series of $g$ diverges unboundedly on $H$. The construction of $T$ then is similar to that in pp. 417-479 of [Ba], starting from that $g$.

It follows from the preceding theorem and its proof that if $C$ is say the Cantor set, and $G \subseteq C$ is a $G_{\delta}$ we can associate to $G$ a continuous function $f \in C(\mathbf{T})$ with

$$
\begin{aligned}
& x \in G \Rightarrow\left\{S_{N}(f, x)\right\} \text { diverges } \\
& x \notin G \Rightarrow f^{\prime}(x) \text { exists. }
\end{aligned}
$$


Moreover there is a Borel function $c \mapsto f_{c}$ such that if $c$ is a code of $G_{\delta}$ subset $G_{c}$ of $C$ then $f_{c}$ is the above function for $G_{c}$. Thus

$$
\begin{aligned}
& G_{c}=\varnothing \Rightarrow f_{c} \in D, \\
& G_{c} \neq \varnothing \Rightarrow f_{c} \notin \mathrm{EC} .
\end{aligned}
$$

Since $P(c) \Leftrightarrow$ " $G_{c}=\varnothing$ " is complete $\Pi_{1}^{1}$ this shows that there is no Borel set $B$ with $D \subseteq B \subseteq$ EC. (One can also easily, as in $\S 3$, translate this into an argument that shows that there is a Borel function $F: 2^{\mathbf{N}^{*}} \rightarrow C(\mathbf{T})$ such that

$$
T \in \mathrm{WF} \Rightarrow F(T) \in D, \quad T \notin \mathrm{WF} \Rightarrow F(T) \notin \mathrm{EC} .)
$$

\section{REFERENCES}

[Ba] N. K. Bari, A treatise on trigonometric series, vols. I, II, Macmillan, New York, 1964.

[Br] A. M. Bruckner, Differentiation of real functions, Lecture Notes in Math., vol. 659, SpringerVerlag, Berlin and New York, 1978.

[Bu1] V. V. Buzdalin, Unbounded divergence of Fourier series of continuous functions, Math. Notes 7 (1970), 5-12. (English translation of Mat. Zametki 7 (1970), 7-18)

[Bu2] - Trigonometric Fourier series of continuous functions diverging on a given set, Math. USSR Sbornik 24 (1974), no. 1, 79-101. (English translation of Mat. Sb. 95(137) (1974), no. 1)

[C] L. Carleson, On convergence and growth of partial sums of Fourier series, Acta Math. 116 (1966), 135-157.

[EHP] P. Erdös, F. Herzog and G. Piranian, Sets of divergence of Taylor series and of trigonometric series, Math. Scand. 2 (1954), 262-266.

[GH] D. C. Gillespie and W. A. Hurewicz, On sequences of continuous functions having continuous limits, Trans. Amer. Math. Soc. 32 (1930), 527-543.

[Ka] Y. Katznelson, An introduction to harmonic analysis, Dover, New York, 1976.

[Ke] A. S. Kechris, Sets of everywhere singular functions, Recursion Theory Week, H.-D. Ebbinghaus et al., ed., Lecture Notes in Math., vol. 1141, Springer-Verlag, Berlin and New York, 1985, pp. 233-244.

[KW] A. S. Kechris and W. H. Woodin, Ranks for differentiable functions, Mathematika (to appear).

[Ku] K. Kuratowski, Evaluation de la classe borélienne ou projective d'un ensemble de points a l'aide des symboles logiques, Fund. Math. 17 (1931), 249-272.

[M] Y. N. Moschovakis, Descriptive set theory, North-Holland, Amsterdam, 1980.

[Ma] R. D. Mauldin, The set of continuous nowhere differentiable functions, Pacific J. Math. 83 (1978), 199-205.

[Maz] S. Mazurkiewicz, Über die Menge der differenzierbaven Functionen, Fund. Math. 27 (1936), 244-249..

[R] W. Rudin, Functional analysis, Tata McGraw-Hill, New Delhi, 1974.

[S1] J. Sladkowska, Sur l'ensemble des points de divergence des series de Fourier des fonctions continues, Fund. Math. 49 (1961), 271-294.

[St] K. R. Stromberg, An introduction to classical real analysis, Wadsworth, Belmont, Calif., 1981.

[Sz] W. Szlenk, The non-existence of a separable reflexive Banach space universal for all separable reflexive Banach spaces, Studia Math. 30 (1968), 53-61.

[Za] A. Zalcwasser, Sur une proprieté du champes des fonctions continus, Studia Math. 2 (1930), 63-67.

[Ze] K. Zeller, Uber Konvergenzmengen von Fourierreihn, Arch. Math. 6 (1955), 335-340.

[Zy] A. Zygmund, Trigonometric series, 2nd ed., Cambridge Univ. Press, 1959.

\section{IBM RESEARCH, SAN JOSE, CALIFORNIA 95193}

Department of Mathematics, California institute of Technology, PasaDENA, CALIFORNIA 01125 